\title{
Keap1-targeting microRNA-941 protects endometrial cells from oxygen and glucose deprivation-re-oxygenation via activation of Nrf2 signaling
}

Shu-ping $\mathrm{Li}^{1+}$, Wei-nan Cheng ${ }^{2+}, \mathrm{Ya} \mathrm{Li}^{3+}$, Hong-bin Xu${ }^{1}$, Hui Han ${ }^{1 *} \mathrm{O}$, Ping $\mathrm{Li}^{4^{*}}$ and Deng-Xia Zhang ${ }^{1 *}$

\begin{abstract}
Background: Mimicking ischemia-reperfusion injury, oxygen and glucose deprivation (OGD)-re-oxygenation (OGDR) applied to endometrial cells produces significant oxidative stress and programmed necrosis, which can be inhibited by nuclear-factor-E2-related factor 2 (Nrf2) signaling. MicroRNA (miRNA)-induced repression of Keap1, a Nrf2 suppressor protein that facilitates Nrf2 degradation, is novel strategy to activate Nrf2 cascade.

Methods: MicroRNA-941 (miR-941) was exogenously expressed in HESC and primary human endometrial cells, and the Nrf2 pathway examined by Western blotting and real-time quantitative PCR analysis. The endometrial cells were treated with OGDR, cell programmed necrosis and apoptosis were tested.

Results: MiR-941 is a novel Keap1-targeting miRNA that regulates Nrf2 activity. In T-HESC cells and primary human endometrial cells, ectopic overexpression of miR-941 suppressed Keap1 3'-UTR (untranslated region) expression and downregulated its mRNA/protein expression, leading to activation of the Nrf2 cascade. Conversely, inhibition of miR-941 elevated Keap1 expression and activity in endometrial cells, resulting in suppression of Nrf2 activation. MiR941 overexpression in endometrial cells attenuated OGDR-induced oxidative stress and programmed necrosis, whereas miR-941 inhibition enhanced oxidative stress and programmed necrosis. MiR-941 overexpression and inhibition were completely ineffective in Keap1-/Nrf2-KO T-HESC cells (using CRISPR/Cas9 strategy). Restoring Keap1 expression, using an UTR-depleted Keap1 construct, abolished miR-941-induced anti-OGDR activity in T-HESC cells. Thus Keap1-Nrf2 cascade activation is required for miR-941-induced endometrial cell protection.

Conclusions: Targeting Keap1 by miR-941 activates Nrf2 cascade to protect human endometrial cells from OGDRinduced oxidative stress and programmed necrosis.
\end{abstract}

Keywords: microRNA-941, Nrf2, Keap1, Endometrial cells, Ischemia-reperfusion injury

\section{Background}

Postpartum hemorrhage is one of the most common complications in obstetric practice [1-3], resulting in ischemia to the endometrium and necrosis of endometrial

\footnotetext{
*Correspondence: drhanhuiey@163.com; lipingksfs7@163.com; 13919255870@163.com

†Shu-ping Li, Wei-nan Cheng and Ya Li contributed equally to this work. 'Obstetrics and Gynecology Department, the Affiliated Changzhou No. 2 People's Hospital of Nanjing Medical University, No. 188 Gehu Lake Road, Wujin District, Changzhou, Jiangsu, China

${ }^{4}$ Department of Radiotherapy and Oncology, Affiliated Kunshan Hospital of Jiangsu University, Suzhou, China

Full list of author information is available at the end of the article
}

cells [1-3]. To study the pathophysiology of ischemic death $[4,5]$, the oxygen and glucose deprivation (OGD)re-oxygenation (OGDR) model is a useful approach to investigate ischemic-reperfusion endometrial cell injury [6-9]. Following reperfusion, endometrial ischemia induces oxidative stress to the endometrial cells [1-3], leading to the accumulation of reactive oxygen species (ROS) and depletion of antioxidants [10-12]. Oxidative injury causes excessive DNA breaks, protein damage and mitochondrial dysfunction [10-12], eventually leading to endometrial cell necrosis [1-3].

(c) The Author(s). 2020 Open Access This article is distributed under the terms of the Creative Commons Attribution 4.0 International License (http://creativecommons.org/licenses/by/4.0/), which permits unrestricted use, distribution, and 
It has been recently shown that cell necrosis can occur via a programmed cell death process [13-16], called "programmed necrosis". In endometrial cells, OGDR promotes p53 translocation to the mitochondria where it forms a complex with the mitochondria permeability transition pore (mPTP) component proteins, cyclophilin-D (CypD) and adenine nucleotide translocator type 1 (ANT1) [17, 18]. This initiates mitochondrial depolarization, mPTP pore opening and cytochrome $\mathrm{C}$ release to cytosol, eventually leading to cell necrosis, but not apoptosis [13-15, 19-22]. We previously reported that CypD inhibition or silencing (by targeted shRNA) suppressed OGDR-induced cytotoxicity and programmed necrosis in endometrial cells [5]. Ginseng Rh2 (GRh2) and keratinocyte growth factor (KGF) also protected endometrial cells from OGDR by shutting down the programmed necrosis pathway $[4,5]$.

The transcription factor nuclear-factor-E2-related factor 2 (Nrf2) promotes the expression of multiple key antioxidant genes and detoxifying enzymes, protecting cells against oxidative injury [23-25]. Nrf2 is localized to the cytoplasm where it binds Kelch-like ECH-associated protein 1 (Keap1) [23-25] and Cul3 ubiquitin ligase, which promotes Nrf2 ubiquitination and proteasomal degradation [23-25]. Activated Nrf2 (i.e. via post-translational modifications) is released from the Keap1-Cul3 complex, leading to Nrf2 protein stabilization [23-25]. Subsequently, Nrf2 translocates to cell nuclei where it binds the antioxidant responsive element (ARE) [23-25], leading to transcription of Nrf2-dependent antioxidant genes [2628]. The majority of the Nrf2-dependent genes, including heme oxygenase-1 (HO-1), NAD(P)H:quinone oxidoreductase 1 (NQO1), $\gamma$-glutamyl cysteine ligase catalytic subunit $(G C L C)$ [23], exert potent antioxidant activity $[29,30]$.

Studies show that forced Nrf2 activation in endometrial cells enhances antioxidant activity and mitigates OGDR-induced oxidative injury. We have previously reported that KGF-induced Nrf2 activation efficiently protected endometrial cells from OGDR [4]. MicroRNAs (miRNAs) are essential and novel players in regulating activation of the Keap1-Nrf2 pathway [31-36]. These 22-nt long single-strand non-coding RNAs (ncRNAs) bind to the $3^{\prime}$ - untranslated region (3'-UTR) of the targeted mRNAs, causing degradation and/or translation repression [37, 38]. A potential strategy to activate the Nrf2 signaling cascade is to reduce Keap1 protein levels by expressing Keap1-targeting miRNAs [31, 35, 36]. Here we show that microRNA-941 ("miR-941") targets Keap1 to activate Nrf2 and protects endometrial cells from OGDR-induced cytotoxicity.

\section{Materials and methods}

\section{Chemical and reagents}

Puromycin and ploybrene were purchased from SigmaAldrich Chemicals Co. (St Louis, Mo). The fluorescent dye JC-1 was provided by Invitrogen Thermo-Fisher (Shanghai, China). All cell culture reagents and qPCR reagents were obtained from Gibco BRL Co. (Grand Island, NY). The antibodies of the present study were provided by Santa Cruz Biotechnology (Santa Cruz, CA) and Cell Signaling Tech (Suzhou, China). The primers, sequences, constructs and virus were designed and provided by Shanghai Genechem Co. (Shanghai, China), unless otherwise motioned.

\section{T-HESC cell culture}

As described previously $[4,5]$, the immortalized human endometrial cell line, T-HESC [39], was cultured in regular DMEM/Hams F-12 nutrient medium plus 10\% FBS.

\section{Culture of primary human endometrial cells}

The fresh human endometrial tissues, acquired from a written-informed consent uterine-bleeding patient (31year old, administrated at Changzhou Second People's Hospital, undergoing the partial hysterectomy surgery) were digested with $0.15 \%$ trypsin-EDTA (Sigma) and Collagenase I (Sigma) for $1 \mathrm{~h}$, and then transferred to DMEM/Hams F-12 nutrient medium plus 15\% FBS. Tissues were dissolved. Blood vessel cells, immune cells and other non-endometrial cells were abandoned through gravity sedimentation. The remaining human endometrial cells were resuspended and cultured in complete DMEM medium [5]. The primary human endometrial cells at passage 3-10 were utilized for biomedical assays. The protocols of using human tissues and cells were approved by the Ethics Committee at Changzhou Second People's Hospital.

\section{miR-941 overexpression or inhibition}

The pre-miR-941 nucleotide sequence and its anti-sense sequence were synthesized and sequence-verified by Shanghai Genechem Co. Each of the two was ligated to the GV248 construct (Shanghai Genechem Co.). The construct, along with the lentivirus-packing helper plasmids (psPAX2 and pMD2.G [33]), were co-transfected to HEK-293 T cells, forming the pre-miR-941-expressing lentivirus ("lv-pre-miR-941") and the pre-miR-941 antisense lentivirus ("antagomiR-941"). Virus were enriched, filtered, and added directly to human endometrial cells (in the polybrene-containing medium). When necessary puromycin $(5.0 \mu \mathrm{g} / \mathrm{mL})$ was included in the medium to select stable cells, with miR-941 levels examined by qPCR.

qPCR

The human endometrial cells, with the applied treatments, were harvest and the total cellular RNA extracted using TRIzol protocol [5]. We utilized an ABI Prism 
7500 Fast Real-Time PCR system to carry out the quantitative real time-PCR (qPCR) assay. To calculate product melting temperature we applied the melt curve analyses. Glyceraldehyde-3-phosphatedehydrogenase (GAPDH) was always examined as the reference gene and the internal control, and the $2^{-\Delta \Delta C t}$ method utilized for the quantification of targeted mRNAs. The following mRNA primers were utilized: NQO1 (NM_000903) forward, 5'-CATTCT GAAAGGCTGGTTTG and reverse, 5'-GGCTGCTTGG AGCAAAATAC; HO1 (NM_002133) forward, 5' GCTACCTGGGTGACCTGTCT and reverse, 5'GGGCAGAATCTTGCACTTTG; Nrf2 (NM_006164) forward, 5'-TGAGCATGCTTCCCATGAT and reverse, 5'-CTTCTCTAGCCGCTCTGTGG; GAPDH (NM 002046) forward, 5 '-CGGAGTCAACGGATTTGGTCGT AT and reverse, 5'-AGCCTTCTCCATGGTGGTGAAG AC. Keap1 (NM_203500) forward: 5'-TACGATGTGG AAACAGAGACGTGGA and reverse 5'-TCAACA GGTACAGTTCTGGTCAATCT. The primers cover exon junction/s, and the amplicons around 90-200 bp. miR-941 was normalized to U6. miR-941 and U6 primers were obtained from OriGene (Beijing, China).

\section{Keap1 3'-UTR activity}

Keap1 3'-UTR reporter plasmid (containing the miR941-binding sites, at position of 276-283) was generated using the same protocol described previously [31], which was transfected to human endometrial cells using the Lipofectamine 2000 protocol. Afterwards, cells were subjected to the applied genetic modifications, with the Keap 13 '-UTR luciferase activity tested through the Promega kit [40].

\section{Transfection of miR-941 mimic}

Human endometrial cells were seeded into the six-well tissue culture plates (at $1 \times 10^{5}$ cells in each well). Lipofectamine 2000 was utilized for the transfection of 500 $\mathrm{nM}$ of the wild-type ("WT") or the mutant ("Mut") miR941 mimics (synthesized by Shanghai Genechem Co.). After 48 h, miR-941 levels were determined by qPCR.

\section{RNA-pull down assay}

The RNA-Pull down assay was carried out through the previously-described protocol [41, 42], testing miR-941bound mRNA using the Pierce Magnetic RNA PullDown Kit, Shanghai, China). In brief, T-HESC cells were transfected with biotinylated miR-941 mimic or control mimic $(100 \mathrm{nmol} / \mathrm{L})$ for $48 \mathrm{~h}$, and cells were harvested using the lysis buffer described early [42]. The biotincaptured RNA complex was pulled down by incubating the cell lysates $(600 \mu \mathrm{g}$ of each treatment) with the streptavidin-coated magnetic beads [41]. The bound mRNA was purified using the RNeasy Mini Kit (QIAGEN, Shanghai, China), with expression of Keap1 $m R N A$, in the bound fractions examined by qPCR. Its levels were normalized to the input controls.

\section{Cell viability}

Human endometrial cells were seeded into the 96-well tissue culture plates (3000 cells in each well). Following the applied treatments, a cell counting kit-8 (CCK-8) kit (Dojindo Laboratories, Kumamoto, Japan) was utilized to examine cell viability [5], with CCK- 8 optic density (OD) examined at test-wavelength of $450 \mathrm{~nm}$.

\section{Lactate dehydrogenase (LDH) assay}

The human endometrial cells were seeded into the 12well tissue culture plates (at $0.5 \times 10^{5}$ cells in each well). LDH release to the cell medium, the quantitative marker of cell necrosis [43], was tested through a two-step LDH detection kit (Promega, Shanghai, China) [5]. LDH contents in the medium were always normalized to total LDH levels.

\section{OGD/re-oxygenation (OGDR)}

As described previously $[4,5]$, human endometrial cells with applied genetic treatments were initially placed into an airtight chamber $\left(95 \% \mathrm{~N}_{2} / 5 \% \mathrm{CO}_{2}\right)$ for $4 \mathrm{~h}$, mimicking OGD. Thereafter, human endometrial cells were returned back to the complete medium and reoxygenated. Cells were further cultured for applied time periods.

\section{Western blotting}

Human endometrial cells were seeded into the six-well tissue culture plates (at $1 \times 10^{5}$ cells in each well). Following the applied treatments, cellular lysates were achieved and quantified [4, 5]. The lysates proteins ( $40 \mu \mathrm{g}$ per treatment into each lane) were separated by SDS-PAGE gels, and transferring to polyvinylidene difluoride (PVDF) blots [44]. The detailed protocols for Western blotting and data quantification (through the Image J software) were previously described [45, 46]. Assaying of nuclear fraction proteins was described early [46].

\section{Mitochondrial immunoprecipitation (Mito-IP)}

T-HESC cells were harvested and resuspended [5], with the supernatants collected as the cytosolic fraction lysates. The pellets were resuspended to achieve mitochondrial fraction lysates and quantified [47]. Mitochondrial fraction lysates $(300 \mu \mathrm{g}$ per treatment) were pre-cleared (using anti-IgG Sepharose beads), and incubated with anti-CypD antibody (Santa Cruz Biotech). The mitochondrial complex was captured by antiIgG. CypD-p53-ANT1 association was examined by Western blotting assaying of CypD-bound proteins. 


\section{JC-1 mitochondrial depolarization assay}

The human endometrial cells were seeded into the 12well tissue culture plates (at $0.5 \times 10^{5}$ cells in each well). In OGDR-stimulated cells with mitochondrial depolarization (" $\Delta \Psi$ ") the JC-1 fluorescent dye shall aggregate, forming green monomers [48]. The detailed protocol of JC-1 protocol was discussed previously [5]. The JC-1 fluorescence intensity was examined at $530 \mathrm{~nm}$ (Titertek Fluoroscan, Germany). The representative JC-1 images, integrating both green and red fluorescence images, were also presented.

\section{Superoxide detection}

Endometrial cells with the applied genetic treatments were initially seeded onto 96-well tissue-culturing plates (at $3 \times 10^{3}$ cells of each well). Following the applied OGDR stimulation, the superoxide colorimetric assay kit (BioVision, Shanghai, China) was applied to examine the cellular superoxide contents. In brief, the superoxide detection reagent $(50 \mu \mathrm{L} /$ well) was added for 15 min under the dark, with the superoxide's absorbance tested at the test-wavelength of $450 \mathrm{~nm}$ [31].

\section{Lipid peroxidation assay}

As reported [31] endometrial cells with the applied genetic treatments were initially seeded onto the six-well tissue-culturing plates (at $1 \times 10^{5}$ cells per well). Following the applied OGDR stimulation, the lipid peroxidation assay kit (Abcam, Shanghai, China) was utilized to examine cellular lipid peroxidation levels, via the malondialdehyde method. The lipid peroxidation levels, determined by the thiobarbituric acid reactive concentration, were tested and quantified using the previouslydescribed protocol $[31,49]$.

\section{NQO1 activity}

The detailed protocol for testing the relative NQO1 activity in human endometrial cells has been described elsewhere [50]. In brief, the inducer potency was quantified by using the NQO1 bioassay. T-HESC cells or the primary human endometrial cells $\left(10^{4}\right.$ per well of a 96 well plate), with applied genetic treatments, were cultured for $24 \mathrm{~h}$. The NQO1 enzyme activity was tested and quantified in cell lysates using menadione as the substrate.

\section{Nrf2 or Keap1 knockout (KO)}

The lentiCRISPR-Nrf2-KO-puro construct and the lentiCRISPR-Keap1-KO-puro construct were described early [31], each was transduced to T-HESC cells (cultured in the polybrene-containing complete medium). Nrf2 sgRNA (Target DNA Sequence: TACACATTCA GCTGGCGCGT, PAM Sequence: AGG) and Keap1 sgRNA (Target DNA Sequence: GTACGCCTCCACTG
AGTGCA, PAM Sequence: AGG) were utilized. The GFP-positive T-HESC cells were sorted via FACS. The selected single cells were further incubated in complete medium with puromycin for 10 days, with Nrf2/Keap1 $\mathrm{KO}$ verified by Western blotting and/or qPCR assays.

\section{Keap1 re-expression}

The Keap1 (with no 3'-UTR region) expression GV248 construct was designed and provided by Shanghai Genechem, transduced to T-HESC cells with miR-941 overexpression. Cells were then selected by puromycin for 10 days, with Keap1 re-expression verified by $\mathrm{QPCR}$ and Western blotting assays.

\section{Statistical analysis}

Data were presented as mean \pm standard deviation (SD). The repeated-measures analysis of variance (RMANOVA) with Dunnett's post hoc test for multiple comparisons (SPSS 16.0, SPSS Co. Chicago, CA) were utilized to evaluate statistical significance. The twotailed unpaired $\mathrm{T}$ test (Excel 2013) was carried out to examine significance between two specific treatment groups. $P<0.05$ was considered statistically significant.

\section{Results}

miR-941 targets Keap1 and activates Nrf2 signaling in human endometrial cells

To identify Keap1-targeting miRNAs the TargetScan (V7.2, http://targetscan.org/) [51] database was first searched. Several potential miRNAs binding to 3 '-UTR of Keap1 were identified and further examined using other miRNA databases, including miRbase (http://www. mirbase.org/) and miRDB (http://www.mirdb.org/ miRDB/policy.html). The prediction algorithms identified miR-941 as a promising candidate that targets the 3'-UTR of Keap1 (at position of 276-283), with context ${ }^{++}$score: -0.59 and the score percentage of $99 \%$ (from TargetScan V7.2 [51], Fig. 1a). Functional studies were performed to validate whether miR-941 could affect Keap1. T-HESC human endometrial cells [4, 5] were transduced with a lentivirus encoding the premicroRNA-941 ("lv-pre-miR-941") and two stable cell lines, "s-L1" and "s-L2", were established. Using qPCR [52], miR-941 levels increased over 20 folds in the lvpre-miR-941-expresing stable cells (Fig. 1b). Significantly, Keap1 mRNA (Fig. 1d) and protein (Fig. 1e) were significantly downregulated, decreasing over $90 \%$ in miR-941-overexpressed cells (Fig. 1c). These results suggest that miR-941 selectively targets and downregulates Keap1 in endometrial T-HESC cells.

Testing the potential effect of miR-941 on Nrf2 signaling, we found that although Nrf2 mRNA levels (Fig. 1f) were unchanged in lv-pre-miR-941-expresing cells, Nrf2 protein levels (Fig. 1e) were significantly elevated (vs. 


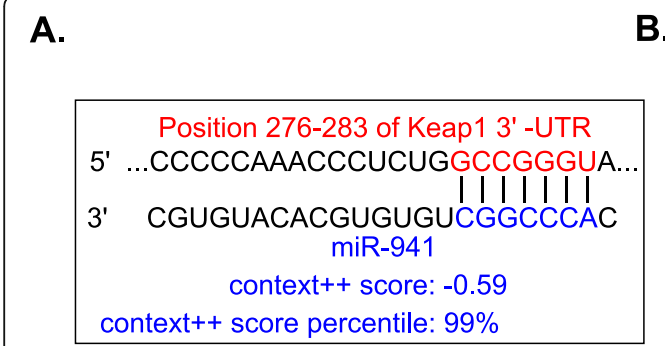

E.

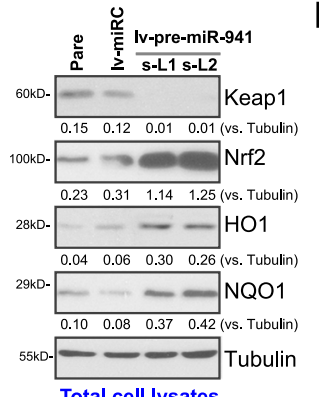

I.

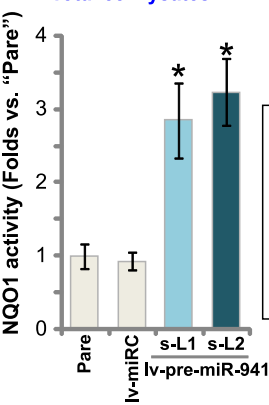

F.

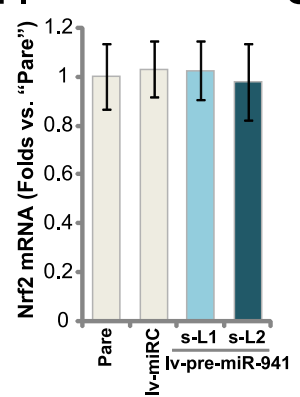

J. miR-941-mimics
B.

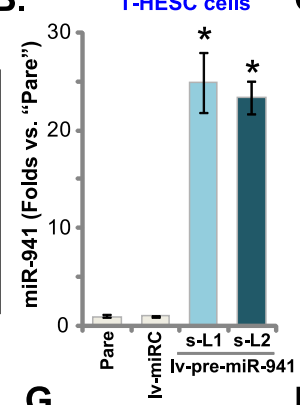

G.

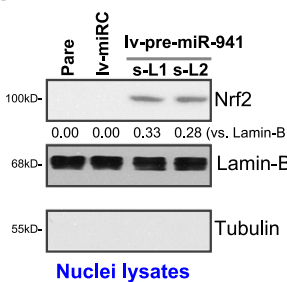

C.

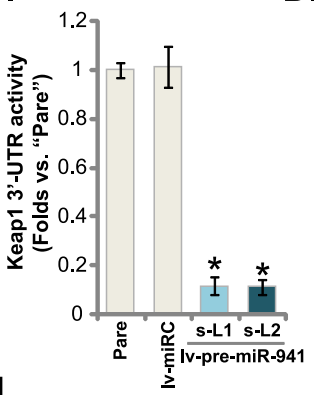

D.
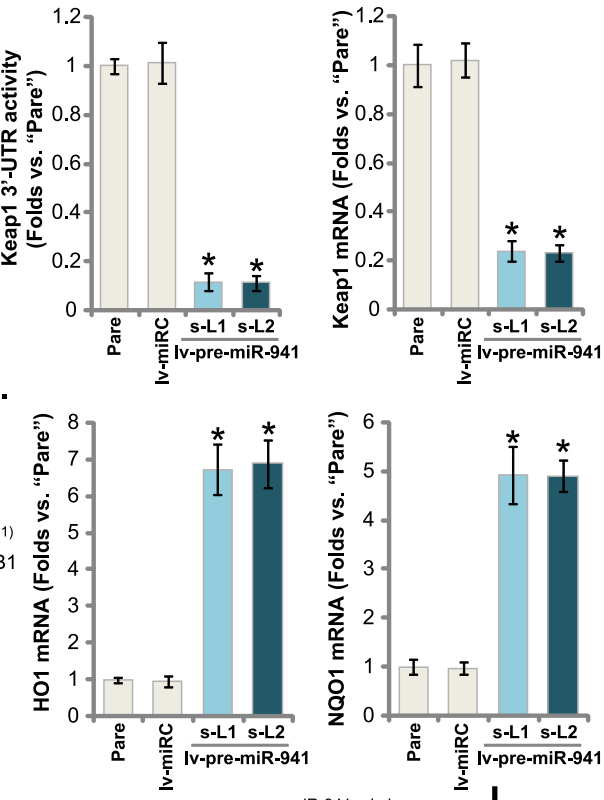

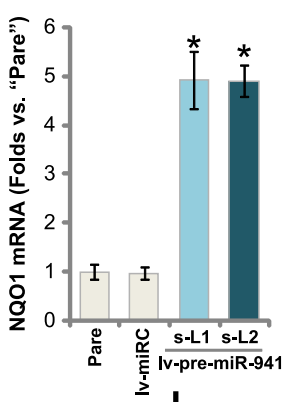

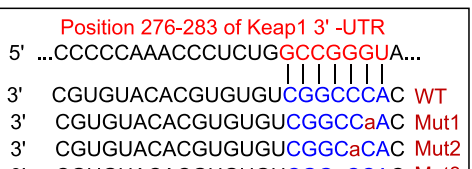
'. CGUGUACACGUGUGUCGGaCCAC Mut3

0.

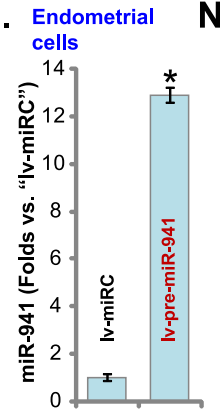

N.

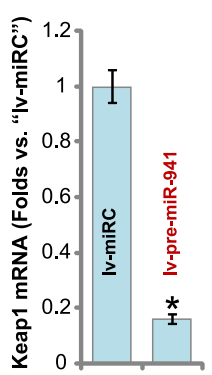

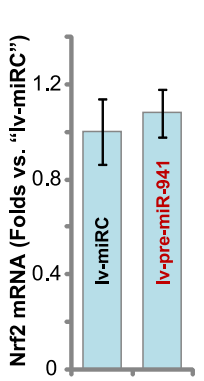

P.

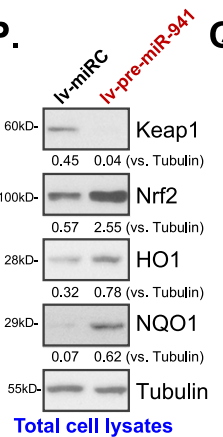

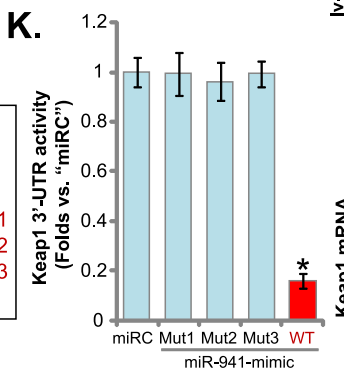
miR-941-mimic

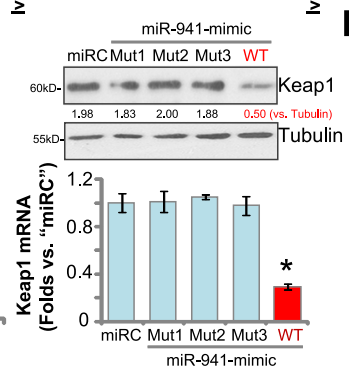

L.

Fig. 1 miR-941 targets Keap1 and activates Nrf2 signaling in human endometrial cells. miRNA-941 putatively targets the 3'-UTR (untranslated region) of human Keap1 mRNA (at position of 276-283) (a). T-HESC human endometrial cells were transduced with lentiviral pre-microRNA-941 ("Iv-pre-miR-941"), with selection by puromycin the stable cells were established, with control cells transduced with lentiviral non-sense microRNA ("Iv-miRC"); Expression of mature miR-941 and listed mRNAs was tested by qPCR assays (b, $\mathbf{d}$, $\mathbf{f}$ and $\mathbf{h}$ ); Keap1 3'-UTR activity was shown (c), with expression of listed proteins in total cell lysates (e) and nuclei lysates ( $\mathbf{g})$ tested by Western blotting; The relative NQO1 activity was tested as well (i). T-HESC cells were transfected with $500 \mathrm{nM}$ of non-sense microRNA control ("miRC"), the wild-type ("WT") or the mutant miR-941 mimics (sequences listed in $\mathbf{j}$ ), with Keap1 3'-UTR activity (k) and Keap1 mRNA/protein expression (k) tested after $48 \mathrm{~h}$. RNA-Pull down assay confirmed the direct association between biotinylated-miR-941 and Keap1 mRNA in T-HESC cells (I). The primary human endometrial cells ("Endometrial cells", same for all Figures) were infected with Iv-pre-miR-941 or Iv-miRC, with expression of listed genes tested by qPCR ( $\mathbf{m}-\mathbf{0}$, and $\mathbf{q}$ ) and Western blotting (p) assays after $48 \mathrm{~h}$. The relative NQO1 activity was tested as well (r). Expression of listed protein was quantified and normalized $(\mathbf{e}, \mathbf{g}, \mathbf{k}$ and $\mathbf{p})$. "Pare" stands for the parental control cells (same for all Figures). Data were presented as mean \pm SD $(n=5)$, and results were normalized. * $\boldsymbol{P}<0.05$ vs. "Iv-miRC"/"miRC" cells. Experiments in this figure were repeated five times with similar results obtained 
control cells). Additionally, the stabilized Nrf2 protein translocated to T-HESC cell nuclei (testing nuclear lysates, Fig. 1g). Furthermore, mRNA and protein expression of two key Nrf2-dependent genes, including $\mathrm{HO} 1$ and NQO1 [33, 53-55], were significantly increased in miR-941-overexpressing cells (Fig. 1e and h), with the NQO1 activity significantly boosted (Fig. 1i). These results show that miR-941 overexpression can induce the Nrf2 signaling cascade activation in T-HESC cells.

To further validate that miR-941 targets Keap1, we transfected into T-HESC endometrial cells with three miR-941miRNAs with mutations in the binding sequence to the Keap1 3'-UTR, "Mut1/2/3" (sequences listed in Fig. 1j). As shown only the wild-type (WT) miR-941 decreased Keap1 3-UTR activity (Fig. 1k, the left panel) and Keap1 $m R N A$ /protein expression (Fig. 1k, the right panel), while the miR-941 mutants were ineffective (Fig. 1k). These results further support that miR-941 targets Keap1 $m R N A$ in T-HESC cells. The nonsense control miRNA ("miRC") exerted no significant effect on Keap1 expression and Nrf2 signaling in T-HESC cells (Fig. 1b-k). To further support our hypothesis the RNA-Pull down assay was performed. As shown (Fig. 11), biotinylated-miR-941 directly associated with Keap1 mRNA (Fig. 1 l).

In the primary human endometrial cells ("Endometrial cells"), infection with lv-pre-miR-941 led to an over 10 fold increase in the expression of mature miR-941 (Fig. 1m), causing downregulation of Keap1 mRNA (Fig. 1n). MiR-941 overexpression similarly induced Nrf2 signaling activation, leading to Nrf2 protein (but not mRNA) accumulation (Fig. 1o and p), HO1 and NQO1 upregulation (both mRNA and protein, Fig. 1p and q) as well as an increase in NQO1 activity (Fig. 1r). Collectively, these results show that miR-941 targets Keap1 and activates Nrf2 signaling in human endometrial cells.

\section{miR-941 overexpression protects human endometrial cells} from OGDR

Our previous studies [4,5] have shown that OGDR mainly induced programmed necrosis, but not apoptosis, in endometrial cells. Here in the control T-HESC cells (with lv-miRC), OGDR similarly induced programmed necrosis, leading to superoxide accumulation and lipid peroxidation (Fig. 2a), mitochondrial CypD-ANT1-p53 association (Fig. 2b), mitochondrial depolarization (tested by JC-1 green fluorescence accumulation, Fig. 2c) and cytosol cytochrome C release (Fig. 2d). Significantly, ectopic overexpression of miR-941, using lv-pre-miR941, potently inhibited OGDR-induced oxidative stress and programmed necrosis in T-HESC cells (Fig. 2a-d). The lv-pre-miR-941-expressing T-HESC cells were protected from OGDR, as indicated by increased cell viability (Fig. 2e) and reduced necrosis (medium LDH release, Fig. 2f), when compared to control cells. Further, T-
HESC cells transfected with the wild-type ("WT") or the miR-941 mutants (see Fig. 1), were subjected to OGDR stimulation. Results show that transfection of the WTmiR-941 potently inhibited OGDR-induced cytotoxicity in T-HESC cells (Fig. 2g and h), while the mutants were completely ineffective (Fig. $2 \mathrm{G}$ and $\mathrm{H}$ ).

In the primary human endometrial cells OGDRinduced ROS production (tested by superoxide accumulation, Fig. 2i), mitochondrial depolarization (Fig. 2j) and cytosol cytochrome $\mathrm{C}$ release (Fig. 2k) were significantly attenuated by ectopic miR-941 overexpression. The latter also alleviated OGDR-induced cytotoxicity in the human endometrial cells (Fig. 2l). These results show that miR-941 overexpression inhibited OGDR-induced oxidative stress, programmed necrosis and cytotoxicity in human endometrial cells.

miR-941 inhibition upregulates Keap1, suppressing Nrf2 signaling in human endometrial cells

To suppress miR-941 expression a pre-microRNA-941 antisense lentivirus ("antagomiR-941") was transduced into T-HESC endometrial cells, and two stable cell lines, "L1" and "L2", established. As compared to control cells transduced with miRNA antisense control lentivirus ("antaC"), mature miR-941 levels decreased by over $90 \%$ in the antagomiR-941-expressing T-HESC cells (Fig. 3a). Significantly, miR-941 inhibition led to a 3-4 fold increase of 3'-UTR activity of Keap1 in T-HESC cells (Fig. 3b), resulting in upregulation of Keap1 mRNA (Fig. 3c) and protein (Fig. 3d) levels. The antagomiR-941 did not affect Nrf2 mRNA levels (Fig. 3e), but did decrease Nrf2 protein levels (Fig. 3d). Furthermore, mRNA (Fig. 3f) and protein (Fig. 3d) expression of HO1-NQO1 as well as the NQO1 activity (Fig. 3g) were significantly decreased in T-HESC cells with antagomiR-941. These results suggest that miR941 is normally involved in the regulation of Keap1 and Nrf2 signaling in T-HESC endometrial cells.

In the primary human endometrial cells antagomiR941 infection similarly downregulated mature miR-941 expression (Fig. 3h), leading to an increase in Keap1 mRNA (Fig. 3i) and protein (Fig. 3j) levels. AntagomiR941 inhibited Nrf2 signaling in the primary human endometrial cells, evidenced by decreased expression of Nrf2 protein (but not Nrf2 mRNA) (Fig. 3j and k), downregulation of HO1-NQO1 (Fig. $3 \mathrm{j}$ and $\mathrm{l}$ ), and reduced NQO1 activity (Fig. 3m). Therefore, miR-941 inhibition upregulates Keap1 but inhibits Nrf2 signaling in primary human endometrial cells.

\section{miR-941 inhibition intensifies OGDR-induced} programmed necrosis in human endometrial cells As miR-941 inhibition suppresses Nrf2 signaling in human endometrial cells, it could intensify OGDR-induced cytotoxicity. To verify this hypothesis, antagomiR-941- 


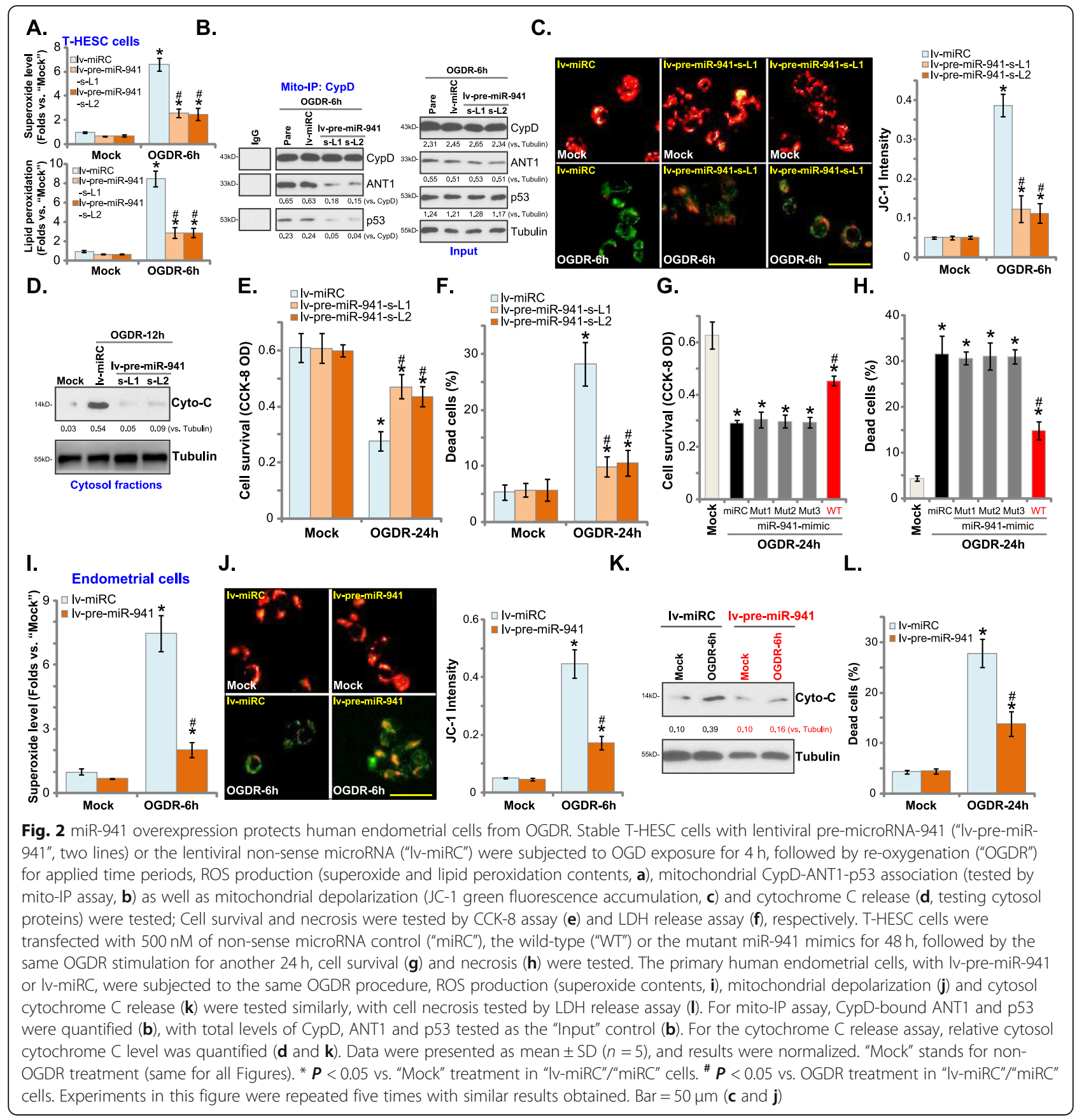

expressing T-HESC cells (Fig. 3) were subjected to OGDR stimulation. As compared to antaC control cells (see Fig. 3), OGDR-induced ROS production tested by superoxide accumulation was significantly increased in antagomiR-941 T-HESC cells (Fig. 4a). Furthermore, OGDR-induced mitochondrial CypD-ANT1-p53 association (Fig. 4b), mitochondrial depolarization (JC-1 green fluorescence accumulation, Fig. 4c) and cytochrome c release to cytosol (Fig. 4d) were augmented bymiR-941 inhibition. Consequently, antagomiR-941 potentiated OGDR-induced necrosis (LDH release to the medium,
Fig. 4e) in T-HESC cells. In the primary human endometrial cells, miR-941 inhibition by antagomiR-941 similarly enhanced OGDR-induced ROS production (Fig. 4f), mitochondrial depolarization (JC-1 green fluorescence accumulation, Fig. 4g) and cytochrome $\mathrm{C}$ release to cytosol (Fig. 4h). Furthermore, OGDR-induced reduced viability (Fig. 4i) and necrosis (Fig. 4j) were exacerbated with miR-941 inhibition in primary endometrial cells. These results demonstrate that miR-941 inhibition intensifies OGDR-induced cytotoxicity in human endometrial cells. 


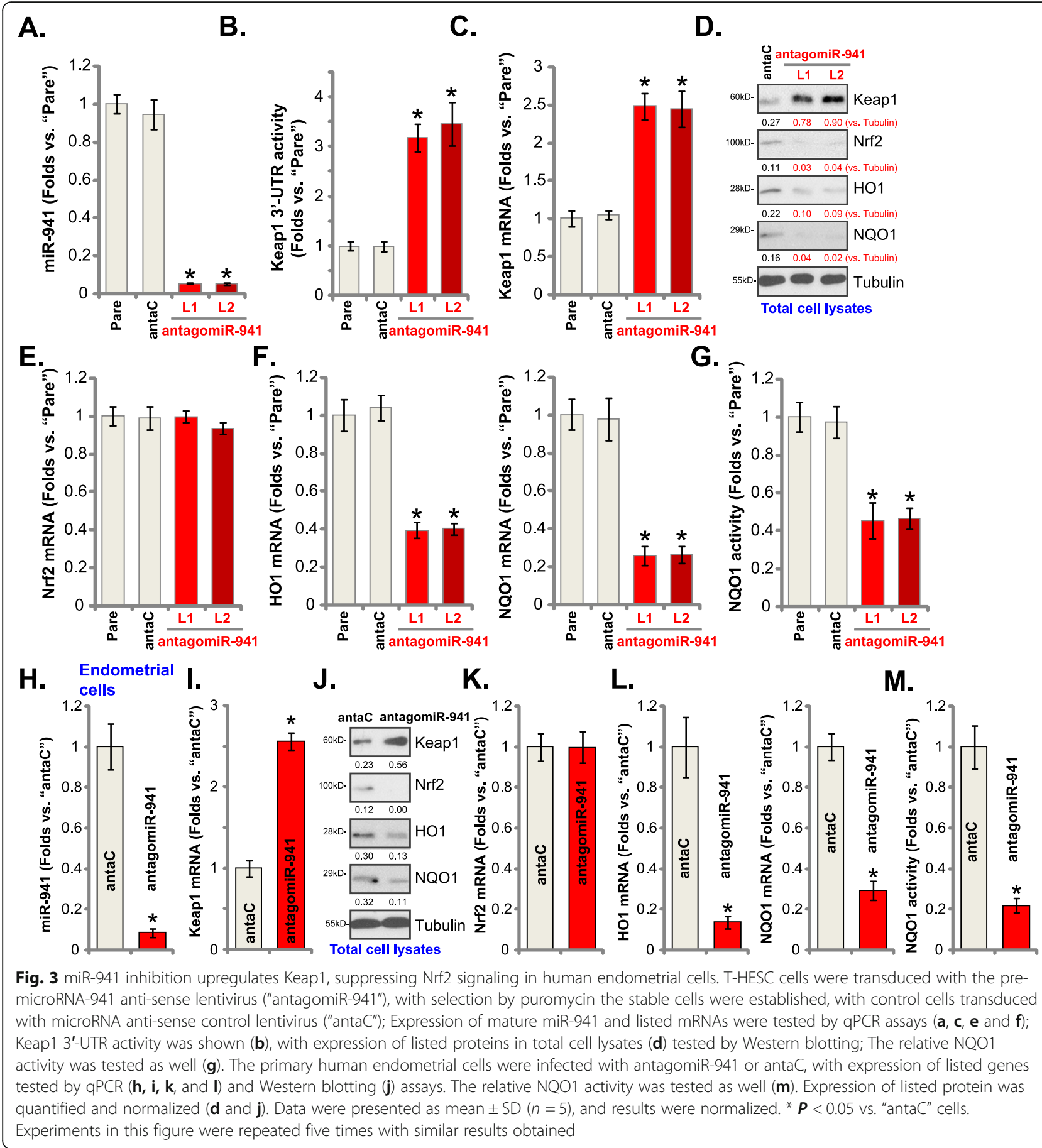

miR-941 fails to affect OGDR-induced cytotoxicity in Keap1/Nrf2-KO human endometrial cells

We tested whether Nrf2 activation is essential for miR941-induced endometrial cell protection against OGDR. Nrf2 knockout (Nrf-KO) T-HESC stable cells were established using a CRISPR/Cas9-Nrf2-KO construct [31], and Nrf2 depletion confirmed by Western blotting (Fig. 5d). Compared to Cas9 control cells ("Cas9-C"), Nrf2-KO cells were more sensitive to OGDR stimulation, showing decreased viability (Fig. 5a) and enhanced cell necrosis (Fig. 5b). Significantly, exogenously altering miR-941 expression, via lv-pre-miR-941 or antagomiR-941 (see qPCR results in Fig. $5 \mathrm{c}$ ), failed to affect OGDR-induced cytotoxicity in Nrf2-KO T-HESC cells (Fig. 5a and b). Nrf2 KO did not affect Keap1 regulation by lv-pre-miR-941 or antagomiR-941, as shown in Fig. 5d. These results suggest that miR-941-induced endometrial cell protection against OGDR requires Nrf2 signaling. 


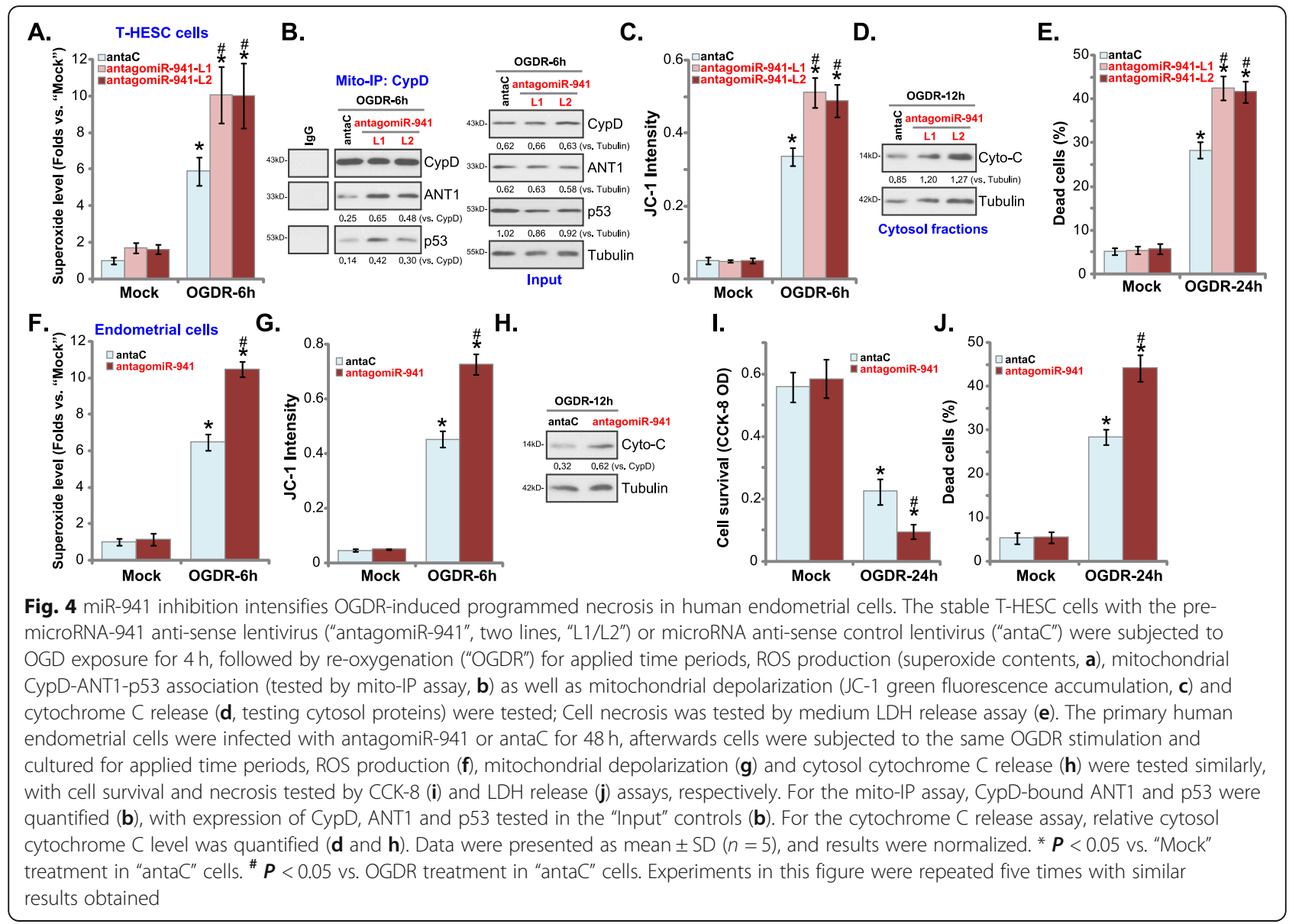

If targeting and silencing Keap1 is the primary mechanism of miR-941-induced endometrial cell protection against OGDR, Keap1 depletion should mimic miR-941 actions. To test this hypothesis, the CRISPR-Cas9 method was applied to knockout Keap1 in T-HESC cells (using a previously described protocol [31]). OGDRinduced cytotoxicity (Fig. 5e) and necrosis (Fig. 5f) were largely attenuated in Keap1-KO T-HESC cells (vs. Cas9$\mathrm{C}$ cells), mimicking the actions of miR-941 overexpression. Importantly, in the Keap1-KO cells, lv-pre-miR941 and antagomiR-941 were both ineffective against OGDR-induced actions (Fig. 5e and f), although both altered miR-941 expression (Fig. 5g). As expected (Fig. 5h), Keap1 depletion in the Keap1-KO T-HESC cells resulted in significant $\mathrm{Nrf} 2$ protein accumulation.

To further support our hypothesis, an UTR-deleted Keap1 construct was transfected into lv-pre-miR-941-expressing T-HESC cells, resulting in Keap1 mRNA (Fig. 5i) and protein (Fig. 5j) re-expression, but Nrf2 protein reduction (Fig. 5j). Functional studies show that reexpression of Keap1 reversed the lv-pre-miR-941-induced T-HESC cell protection against OGDR, leading to cell necrosis (Fig. 5k). Notably, Keap1 re-expression did not affect miR-941 overexpression in lv-pre-miR-941-T-
HESC cells (Fig. 5l). These results further support that Keap1-Nrf2 cascade activation is essential for miR-941induced endometrial cell protection against OGDR.

\section{Discussion}

The expression and potential function of miR-941 in human endometrial cells is unknown. The study by $\mathrm{Hu}$ et al., has shown that miR-941 is a human-specific miRNA, highly expressed in the human brain and in other human cells [56]. It has regulatory effects on gene expression, participating in hedgehog- and insulinsignaling pathways [56]. Zhang et al., have shown that miR-941 levels are significantly downregulated in hepatocellular carcinoma (HCC) tissues. Functionally, miR941 negatively regulated KDM6B (lysine (K)-specific demethylase $6 \mathrm{~B}$ ) to inhibit HCC cell epithelialmesenchymal transition (EMT) and cell migration/invasion [57]. Bai et al., reported that miR-941 levels are significantly higher in acute coronary syndrome patients than those in healthy controls [58].

Recent studies demonstrate that miRNA-induced silencing of Keap1, the Nrf2 suppressor protein, is a novel strategy to regulate Nrf2activation in human cells. In breast cancer cells miR-200a targeted Keap1 to activate 


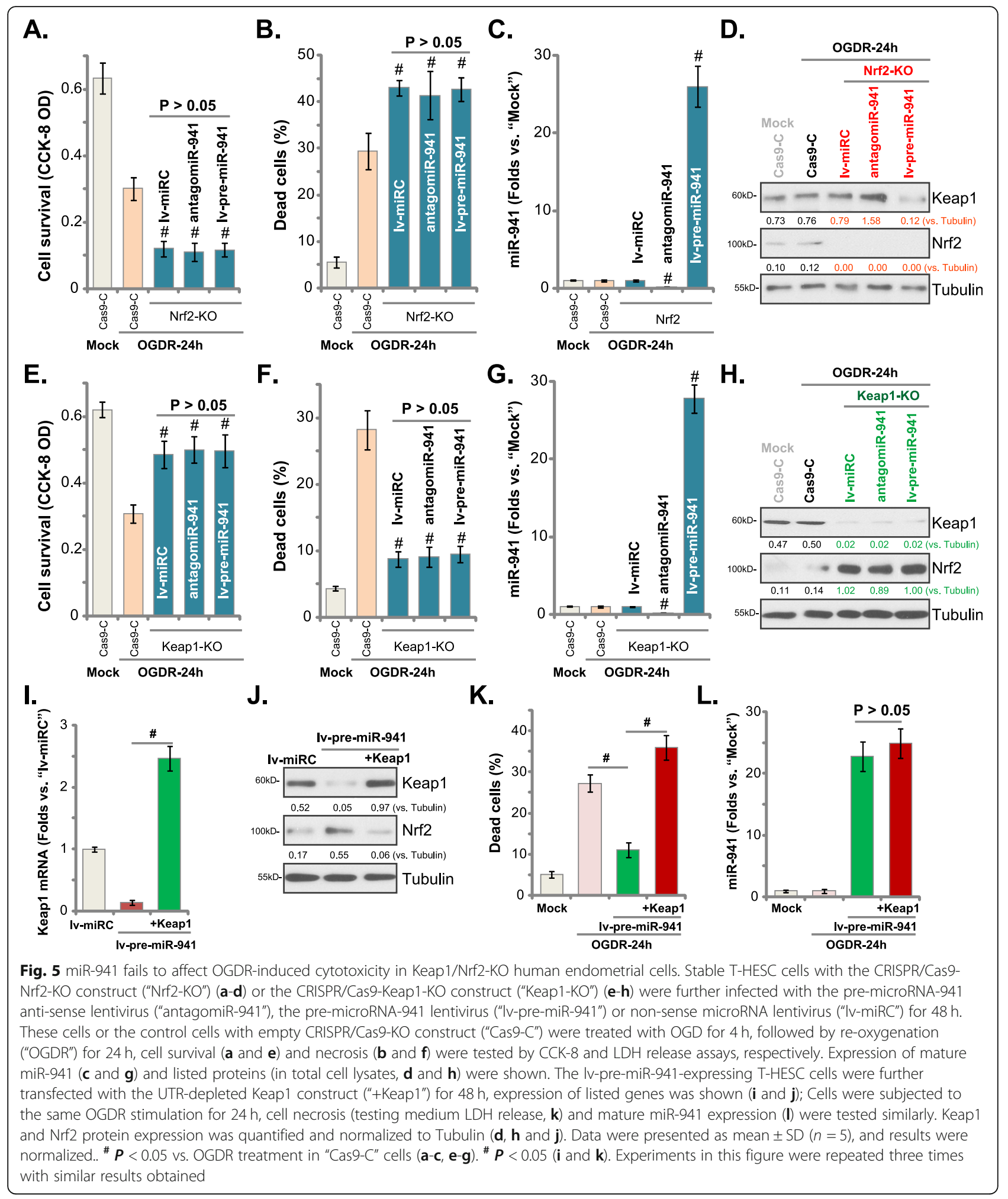

Nrf2 signaling cascade [36]. In SH-SY5Y neuroblastoma cells and differentiated human neural progenitor cells, miR-7 activated Nrf2 signaling by targeting and silencing Keap1 [35]. In retinal pigment epithelium cells (RPEs) and retinal ganglion cells (RGCs), miR-141 activated Nrf2 signaling by targeting miR-141, protecting cells against ultra-violet (UV)-induced oxidative stress [59]. A recent study by $\mathrm{Xu}$ et al., has shown that Keap1- 
targeting miR-626 activated Nrf2 signaling and protected RPEs from oxidative injury [31]. Therefore, Keap1targeting miRNAsinduceNrf2 activation to protect human cells from oxidative stress.

The results of this study confirm that miR-941 is a direct and specific Keap1-targeting miRNA, that regulates the Keap1-Nrf2 cascade in human endometrial cells. In T-HESC cells and primary human endometrial cells forced miR-941 overexpression inhibited Keap1 3'-UTR reporter luciferase activity and downregulated Keap1 mRNA/protein expression, subsequently leading to Nrf2 activation. Conversely, Keap1 3'-UTR reporter luciferase activity and expression were elevated in endometrial cells with miR-941 inhibition, whereas Nrf2 activation was inhibited. RNA-Pull down experiments confirmed that miR-941 directly binds Keap1 mRNA in T-HESC cells. Functional studies confirmed that miR-941 exerted significant endometrial cytoprotection against OGDR. Ectopic miR-941 overexpression in endometrial cells largely inhibited OGDR-induced ROS production and programmed necrosis. Thus, miR-941 promotes Nrf2 cascade activation by targeting and silencing Keap1. Therefore, miR-941 expression provides a novel strategy to protect human endometrial cells from OGDR and possible ischemia-reperfusion injuries.

Our results support that activation of the Keap1-Nrf2 cascade is absolutely required for miR-941-induced endometrial cell protection. Nrf2 KO, using the CRISPR/Cas9 method, abolished miR-941-induced endometrial cytoprotection against OGDR. Furthermore, CRISPR/Cas9-induced Keap1 KO mimicked miR-941 actions and largely attenuated OGDR-induced cytotoxicity in T-HESC cells. Ectopic miR-941 overexpression and miR-941 inhibition were both ineffective against OGDR in the Nrf2-KO and Keap1-KO T-HESC cells. Significantly, restoring Keap1 expression, using an UTR-depleted Keap1 construct, abolished miR-941-induced endometrial cytoprotection against OGDR. Furthermore, transfection of the miR-941 mimics, with mutations at the binding sites to Keap1's 3'UTR, failed to protect endometrial cells from OGDR. These results demonstrate that in endometrial cells miR941-induced activation of the Keap1-Nrf2 cascade is responsible for protection against OGDR.

\section{Conclusion}

MiR-941 negatively regulates Keap1 to activate Nrf2 signaling cascade, thus protecting human endometrial cells from OGDR-induced oxidative stress and programmed necrosis.

\section{Abbreviations}

ANT1: Adenine nucleotide translocator type 1; ARE: Antioxidant responsive element; CCK-8: Cell counting kit-8; CypD: Cyclophilin-D; GCLC: Y-glutamyl cysteine ligase catalytic subunit; GRh2: Ginseng Rh2; HO-1: Heme oxygenase1; Keap1: Kelch-like ECH-associated protein 1; KGF: Keratinocyte growth factor; KO: Knockout; LDH: Lactate dehydrogenase; miR-941: MicroRNA-941; miRNA: MicroRNA; Mito-IP: Mitochondrial immunoprecipitation; mPTP: Mitochondria permeability transition pore; ncRNAs: Non-coding RNAs; NQO1: NAD(P)H:quinone oxidoreductase 1; Nrf2: Nuclear-factor-E2-related factor 2; OD: Optic density; OGD: Oxygen and glucose deprivation; OGDR: OGD-re-oxygenation; qPCR: Quantitative real time-PCR; SD: Standard deviation; UTR: Untranslated region

\section{Acknowledgements}

We thank Dr. Cao from Soochow University for the manuscript editing and study design.

\section{Authors' contributions}

All listed authors designed the study, performed the experiments and the statistical analysis, and wrote the manuscript. All authors have read the manuscript and approved the final version.

\section{Authors' information}

Not applicable.

\section{Funding}

This project was supported by the Fund of Suzhou Municipal Health Bureau, and Fund of Changzhou Municipal Health Bureau, by Kunshan Science and Technology Program (KS18057), Jiangsu University Clinical Medical Science and Technology Development Fund 2018 (JLY20180012) and by Suzhou Science and Technology plan project (KJXW2019064). The funders had no role in the study design, data collection and analysis, decision to publish, or preparation of the manuscript.

\section{Availability of data and materials}

All data generated during this study are included in this published article. Data will be made available upon request.

Ethics approval and consent to participate

This study was approved by the Ethics Committee of Soochow University.

\section{Consent for publication}

Not applicable.

\section{Competing interests}

The authors declare that they have no competing interests.

\section{Author details}

'Obstetrics and Gynecology Department, the Affiliated Changzhou No. 2 People's Hospital of Nanjing Medical University, No. 188 Gehu Lake Road, Wujin District, Changzhou, Jiangsu, China. ${ }^{2}$ Department of Orthopedics, The First Affiliated Hospital of Xiamen University, Xiamen, China. ${ }^{3}$ The Central Lab, North District, Suzhou Municipal Hospital affiliated to Nanjing Medical University, Suzhou, China. ${ }^{4}$ Department of Radiotherapy and Oncology, Affiliated Kunshan Hospital of Jiangsu University, Suzhou, China.

Received: 22 November 2019 Accepted: 29 January 2020

\section{References}

1. Mullins TL, Miller RJ, Mullins ES. Evaluation and Management of Adolescents with abnormal uterine bleeding. Pediatr Ann. 2015;44:e218-22.

2. Van de Velde M, Diez C, Varon AJ. Obstetric hemorrhage. Curr Opin Anaesthesiol. 2015;28:186-90.

3. Deering S, Rowland J. Obstetric emergency simulation. Semin Perinatol. 2013;37:179-88.

4. Shi X, Liu HY, Li SP, Xu HB. Keratinocyte growth factor protects endometrial cells from oxygen glucose deprivation/re-oxygenation via activating Nrf2 signaling. Biochem Biophys Res Commun. 2018;501:178-85.

5. Tang XF, Liu HY, Wu L, Li MH, Li SP, Xu HB. Ginseng Rh2 protects endometrial cells from oxygen glucose deprivation/re-oxygenation. Oncotarget. 2017;8:105703-13.

6. Zheng K, Zhang Q, Lin G, Li Y, Sheng Z, Wang J, Chen L, Lu HH. Activation of Akt by SC79 protects myocardiocytes from oxygen and glucose deprivation (OGD)/re-oxygenation. Oncotarget. 2017;8:14978-87. 
7. Zhao H, Mitchell S, Ciechanowicz S, Savage S, Wang T, Ji X, Ma D. Argon protects against hypoxic-ischemic brain injury in neonatal rats through activation of nuclear factor (erythroid-derived 2)-like 2. Oncotarget. 2016;7: 25640-51.

8. Gu DM, Lu PH, Zhang K, Wang X, Sun M, Chen GQ, Wang Q. EGFR mediates astragaloside IV-induced Nrf2 activation to protect cortical neurons against in vitro ischemia/reperfusion damages. Biochem Biophys Res Commun. 2015:457:391-7.

9. Almeida A, Delgado-Esteban M, Bolanos JP, Medina JM. Oxygen and glucose deprivation induces mitochondrial dysfunction and oxidative stress in neurones but not in astrocytes in primary culture. J Neurochem. 2002;81:207-17.

10. Ferrari RS, Andrade CF. Oxidative stress and lung ischemia-reperfusion injury. Oxidative Med Cell Longev. 2015;2015:590987.

11. Kalogeris T, Bao Y, Korthuis RJ. Mitochondrial reactive oxygen species: a double edged sword in ischemia/reperfusion vs preconditioning. Redox Biol. 2014;2:702-14.

12. Sanderson TH, Reynolds CA, Kumar R, Przyklenk K, Huttemann M. Molecular mechanisms of ischemia-reperfusion injury in brain: pivotal role of the mitochondrial membrane potential in reactive oxygen species generation. Mol Neurobiol. 2013;47:9-23.

13. Zhang LY, Wu YL, Gao XH, Guo F. Mitochondrial protein cyclophilin-Dmediated programmed necrosis attributes to berberine-induced cytotoxicity in cultured prostate cancer cells. Biochem Biophys Res Commun. 2014;450: 697-703.

14. Qin LS, Jia PF, Zhang ZQ, Zhang SM. ROS-p53-cyclophilin-D signaling mediates salinomycin-induced glioma cell necrosis. J Exp Clin Cancer Res. 2015:34:57.

15. Ju T, Gao D, Fang ZY. Targeting colorectal cancer cells by a novel sphingosine kinase 1 inhibitor PF-543. Biochem Biophys Res Commun. 2016; 470:728-34.

16. Guo F, Liu SQ, Gao XH, Zhang LY. AICAR induces AMPK-independent programmed necrosis in prostate cancer cells. Biochem Biophys Res Commun. 2016;474:277-83.

17. Bonora M, Pinton P. The mitochondrial permeability transition pore and cancer: molecular mechanisms involved in cell death. Front Oncol. 2014;4:302.

18. Javadov S, Kuznetsov A. Mitochondrial permeability transition and cell death: the role of cyclophilin d. Front Physiol. 2013;4:76.

19. Chen B, Xu M, Zhang H, Wang JX, Zheng P, Gong L, Wu GJ, Dai T. Cisplatininduced non-apoptotic death of pancreatic cancer cells requires mitochondrial cyclophilin-D-p53 signaling. Biochem Biophys Res Commun. 2013:437:526-31.

20. Halestrap AP, Gillespie JP, O'Toole A, Doran E. Mitochondria and cell death: a pore way to die? Symp Soc Exp Biol. 2000;52:65-80.

21. Halestrap AP. Calcium, mitochondria and reperfusion injury: a pore way to die. Biochem Soc Trans. 2006:34:232-7.

22. Halestrap A. Biochemistry: a pore way to die. Nature. 2005;434:578-9.

23. Suzuki T, Yamamoto M. Molecular basis of the Keap1-Nrf2 system. Free Radic Biol Med. 2015;88:93-100.

24. Li W, Kong AN. Molecular mechanisms of Nrf2-mediated antioxidant response. Mol Carcinog. 2009;48:91-104.

25. Itoh K, Tong Kl, Yamamoto M. Molecular mechanism activating Nrf2-Keap1 pathway in regulation of adaptive response to electrophiles. Free Radic Biol Med. 2004;36:1208-13.

26. Schmidlin CJ, Dodson MB, Madhavan L, Zhang DD. Redox regulation by NRF2 in aging and disease. Free Radic Biol Med. 2019:134:702-7.

27. Krajka-Kuzniak V, Paluszczak J, Baer-Dubowska W. The Nrf2-ARE signaling pathway: an update on its regulation and possible role in cancer prevention and treatment. Pharmacol Rep. 2017;69:393-402.

28. Sporn MB, Liby KT. NRF2 and cancer: the good, the bad and the importance of context. Nat Rev Cancer. 2012:12:564-71.

29. Wardyn JD, Ponsford AH, Sanderson CM. Dissecting molecular cross-talk between Nrf2 and NF-kappaB response pathways. Biochem Soc Trans. 2015:43:621-6.

30. Kundu JK, Surh YJ. Nrf2-Keap1 signaling as a potential target for chemoprevention of inflammation-associated carcinogenesis. Pharm Res. 2010;27:999-1013.

31. Xu XZ, Tang Y, Cheng LB, Yao J, Jiang Q, Li KR, Zhen YF. Targeting Keap1 by miR-626 protects retinal pigment epithelium cells from oxidative injury by activating Nrf2 signaling. Free Radic Biol Med. 2019;143:387-96.

32. Sun GL, Huang D, Li KR. Jiang Q: microRNA-4532 inhibition protects human lens epithelial cells from ultra-violet-induced oxidative injury via activating SIRT6-Nrf2 signaling. Biochem Biophys Res Commun. 2019;514:777-84.
33. Chen ZJ, Rong L, Huang D, Jiang Q. Targeting cullin 3 by miR-601 activates Nrf2 signaling to protect retinal pigment epithelium cells from hydrogen peroxide. Biochem Biophys Res Commun. 2019;515:679-87.

34. Zhao S, Mao L, Wang SG, Chen FL, Ji F, Fei HD. MicroRNA-200a activates Nrf2 signaling to protect osteoblasts from dexamethasone. Oncotarget. 2017:8:104867-76.

35. Kabaria S, Choi DC, Chaudhuri AD, Jain MR, Li H, Junn E. MicroRNA-7 activates Nrf2 pathway by targeting Keap1 expression. Free Radic Biol Med. 2015;89:548-56

36. Eades G, Yang M, Yao Y, Zhang Y, Zhou Q. miR-200a regulates Nrf2 activation by targeting Keap1 mRNA in breast cancer cells. J Biol Chem. 2011;286:40725-33.

37. Du H, Guo L, Fang F, Chen D, Sosunov AA, McKhann GM, Yan Y, Wang C, Zhang H, Molkentin JD, et al. Cyclophilin D deficiency attenuates mitochondrial and neuronal perturbation and ameliorates learning and memory in Alzheimer's disease. Nat Med. 2008;14:1097-105.

38. Huang AL, Ostrowski MC, Berard D, Hager GL. Glucocorticoid regulation of the ha-MuSV p21 gene conferred by sequences from mouse mammary tumor virus. Cell. 1981:27:245-55.

39. Annunziata M, Grande C, Scarlatti F, Deltetto F, Delpiano E, Camanni M, Ghigo E, Granata R. The growth hormone-releasing hormone (GHRH) antagonist JV-1-36 inhibits proliferation and survival of human ectopic endometriotic stromal cells (ESCs) and the T HESC cell line. Fertil Steril. 2010;94:841-9.

40. Liu YY, Chen MB, Cheng L, Zhang ZQ, Yu ZQ, Jiang Q, Chen G. Cao C: microRNA-200a downregulation in human glioma leads to Galphai1 overexpression, Akt activation, and cell proliferation. Oncogene. 2018;37:2890-902.

41. Wang R, Zhang S, Chen X, Li N, Li J, Jia R, Pan Y, Liang H. CircNT5E acts as a sponge of miR-422a to promote Glioblastoma tumorigenesis. Cancer Res. 2018;78:4812-25.

42. Wang K, Long B, Liu F, Wang JX, Liu CY, Zhao B, Zhou LY, Sun T, Wang M, Yu T, et al. A circular RNA protects the heart from pathological hypertrophy and heart failure by targeting miR-223. Eur Heart J. 2016;37:2602-11.

43. Xie J, Li Q, Ding X, Gao Y. GSK1059615 kills head and neck squamous cell carcinoma cells possibly via activating mitochondrial programmed necrosis pathway. Oncotarget. 2017:8:50814-23.

44. Cao C, Rioult-Pedotti MS, Migani P, Yu CJ, Tiwari R, Parang K, Spaller MR, Goebel DJ, Marshall J. Impairment of TrkB-PSD-95 signaling in Angelman syndrome. PLoS Biol. 2013;11:e1001478.

45. Cao C, Huang $X$, Han Y, Wan Y, Birnbaumer L, Feng GS, Marshall J, Jiang M, Chu WM. Galpha(i1) and Galpha(i3) are required for epidermal growth factormediated activation of the Akt-mTORC1 pathway. Sci Signal. 2009;2:ra17.

46. Zhang H, Liu YY, Jiang Q, Li KR, Zhao YX, Cao C, Yao J. Salvianolic acid a protects RPE cells against oxidative stress through activation of Nrf2/HO-1 signaling. Free Radic Biol Med. 2014;69:219-28.

47. Zhen YF, Wang GD, Zhu LQ, Tan SP, Zhang FY, Zhou XZ, Wang XD. P53 dependent mitochondrial permeability transition pore opening is required for dexamethasone-induced death of osteoblasts. J Cell Physiol. 2014;229: 1475-83.

48. Brooks MM, Neelam S, Fudala R, Gryczynski I, Cammarata PR. Lenticular mitoprotection. Part a: monitoring mitochondrial depolarization with JC-1 and artifactual fluorescence by the glycogen synthase kinase-3beta inhibitor, SB216763. Mol Vis. 2013:19:1406-12.

49. Liu WY, Liou SS, Hong TY, Liu IM. Protective effects of hesperidin (Citrus Flavonone) on high glucose induced oxidative stress and apoptosis in a cellular model for diabetic retinopathy. Nutrients. 2017;9:E1312.

50. Mills EL, Ryan DG, Prag HA, Dikovskaya D, Menon D, Zaslona Z, Jedrychowski MP, Costa ASH, Higgins M, Hams E, et al. Itaconate is an antiinflammatory metabolite that activates Nrf2 via alkylation of KEAP1. Nature. 2018:556:113-7.

51. Agarwal V, Bell GW, Nam JW, Bartel DP. Predicting effective microRNA target sites in mammalian mRNAs. Elife. 2015:4.

52. Svendsen JB, Baslund B, Cramer EP, Rapin N, Borregaard N, Cowland JB. MicroRNA-941 expression in Polymorphonuclear granulocytes is not related to Granulomatosis with Polyangiitis. PLoS One. 2016;11:e0164985.

53. Tang C, Tan S, Zhang Y, Dong L, Xu Y. Activation of Keap1-Nrf2 signaling by 4-octyl itaconate protects human umbilical vein endothelial cells from high glucose. Biochem Biophys Res Commun. 2019;508:921-7.

54. Liu H, Feng Y, Xu M, Yang J, Wang Z, Di G. Four-octyl itaconate activates Keap1-Nrf2 signaling to protect neuronal cells from hydrogen peroxide. Cell Commun Signal. 2018;16:81. 
55. Li C, Yan K, Wang W, Bai Q, Dai C, Li X, Huang D. MIND4-17 protects retinal pigment epithelium cells and retinal ganglion cells from UV. Oncotarget. 2017:8:89793-801.

56. Hu HY. He L, Fominykh K, Yan Z, Guo S, Zhang X, Taylor MS, Tang L, Li J, Liu $J$, et al: evolution of the human-specific microRNA miR-941. Nat Commun. 2012;3:1145.

57. Zhang PP, Wang XL, Zhao W, Qi B, Yang Q, Wan HY, Shuang ZY, Liu M, Li X, Li S, Tang H. DNA methylation-mediated repression of miR-941 enhances lysine (K)-specific demethylase 6B expression in hepatoma cells. J Biol Chem. 2014;289:24724-35.

58. Bai R, Yang Q, Xi R, Li L, Shi D. Chen K: miR-941 as a promising biomarker for acute coronary syndrome. BMC Cardiovasc Disord. 2017;17:227.

59. Cheng LB, Li KR, Yi N, Li XM, Wang F, Xue B, Pan YS, Yao J, Jiang Q, Wu ZF. miRNA-141 attenuates UV-induced oxidative stress via activating Keap1-Nrf2 signaling in human retinal pigment epithelium cells and retinal ganglion cells. Oncotarget. 2017:8:13186-94.

\section{Publisher's Note}

Springer Nature remains neutral with regard to jurisdictional claims in published maps and institutional affiliations.

Ready to submit your research? Choose BMC and benefit from:

- fast, convenient online submission

- thorough peer review by experienced researchers in your field

- rapid publication on acceptance

- support for research data, including large and complex data types

- gold Open Access which fosters wider collaboration and increased citations

- maximum visibility for your research: over $100 \mathrm{M}$ website views per year

At BMC, research is always in progress.

Learn more biomedcentral.com/submissions 